\title{
Metaphysics of science as naturalized metaphysics
}

\author{
Michael Esfeld \\ University of Lausanne, Department of Philosophy \\ Michael-Andreas.Esfeld@unil.ch
}

(for Anouk Barberousse, Denis Bonnay and Mikaël Cozic (eds.): The philosophy of science. A companion. Oxford: Oxford University Press 2018, pp. 142-170.)

\begin{abstract}
This chapter outlines a metaphysics of science in the sense of a naturalized metaphysics. It considers in the first place the interplay of physics and metaphysics in Newtonian mechanics, then goes into the issues for the metaphysics of time that relativity physics raises, shows that what one considers as the referent of quantum theory depends on metaphysical considerations and finally explains how the stance that one takes with respect to objective modality and laws of nature shapes the options that are available for an ontology of quantum physics. In that way, this chapter seeks to make a case for a natural philosophy that treats physics and metaphysics as inseparable in the enquiry into the constitution of the world, there being neither a neo-positivist way of deducing metaphysics from the formalisms of physical theories, nor a neo-rationalist realm of investigation for metaphysics that is independent of physics.
\end{abstract}

\section{What is metaphysics of science?}

Metaphysics of science is a metaphysics because it puts forward ontological claims (that is, claims about what there is in the world) by basing itself on science - instead of conceptual analysis, common sense, or intuitions. By "metaphysics", one does in this context not mean a theory that claims to refer to a domain of being beyond the empirical realm, but, in the Aristotelian sense, a theory that seeks to achieve a general and fundamental understanding of the empirical world itself (see Aristotle, Metaphysics, book 4). Today's metaphysics of science is considered as being part and parcel of analytic philosophy broadly conceived, which, since its metaphysical turn, no longer focuses on the analysis of language. It is instead a systematic and argumentative enterprise that seeks to achieve a comprehensive view of the world and our place in it - in short, pursuing what philosophy has been since its beginnings in Plato and Aristotle. The trait that distinguishes metaphysics of science from standard analytic philosophy is its being anchored in science: one bases oneself on science in doing metaphysics. It is therefore common today to use the term naturalized metaphysics.

Indeed, standard analytic metaphysics seeks to find out truth about the constitution of the world mainly based on conceptual analysis linked with common sense realism and intuitions. This enterprise is not hostile to science: a global supervenience thesis to the effect that everything that there is in the world supervenes on a basis that is investigated by fundamental physics is common ground. But standard analytic metaphysics does not show any particular interest in research about what this basis may be like given our current fundamental physical theories. Jackson's (1998) plea for conceptual analysis is a good illustration of this situation.

Naturalized metaphysics is opposed to the methodology employed by this type of metaphysics. The term "naturalized metaphysics" characterizes not so much the ontological 
stance of naturalism - a stance shared by most adherents to conceptual analysis -, but the method that seeks to find out truth about the constitution of the world by means of a close examination of our current fundamental physical theories. In particular, any metaphysical claim is to be motivated and justified by the content of our best scientific theories - by contrast to conceptual analysis, common sense, or intuitions. The book by Ladyman and Ross (2007) is the most forceful articulation of this type of metaphysics. ${ }^{1}$

This opposition in methodology does not necessarily lead to an opposition in the content of the metaphysics thus obtained, given in particular the nearly universal acceptance of the mentioned global supervenience thesis. However, naturalized metaphysics often comes with a good deal of polemic against analytic metaphysics: it is suggested that the best candidate for truth about the constitution of the world that we can currently achieve can be read from our fundamental physical theories and that what thus can be extracted from physics contradicts much of what is commonly accepted in standard metaphysics.

This chapter investigates how metaphysics of science qua naturalized metaphysics can work when taking fundamental physics as a guideline. To start with, I will consider the ontology of Newtonian mechanics (section 2), followed by an enquiry into the special theory of relativity and its alleged philosophical consequences for the metaphysics of time (section 3). I will then go into the options for an ontology of quantum physics (section 4) and examine how these options depend on the stance that one takes with respect to laws of nature and modality (section 5). By contrast to what Ladyman and Ross (2007) suggest, it will become increasingly clear during this investigation that there is no one-way road from physics to metaphysics, but that any ontology of physics has to bring in both the physical theory in question and considerations from standard metaphysics. ${ }^{2}$ In a nutshell, there neither is a neopositivist way of deducing metaphysics from physics, nor a neo-rationalist realm of investigation for metaphysics that is independent of physics. What we need is a metaphysics of science or a naturalized metaphysics that is a natural philosophy as practised in the $17^{\text {th }}$ and $18^{\text {th }}$ century, when physics and metaphysics were treated as forming a seamless whole.

\section{Newton's natural philosophy}

Classical mechanics proposes an ontology of matter in motion: the fundamental physical domain consists in moving particles, with the laws of nature accounting for the way in which the particles move. ${ }^{3}$ Thus, Newton famously writes at the end of the "Opticks" (1704):

... it seems probable to me, that God in the Beginning form'd Matter in solid, massy, hard, impenetrable, moveable Particles ... the Changes of corporeal Things are to be placed only in the various Separations and new Associations and motions of these permanent Particles.

(Question 31,p. 400 in the edition Newton 1952)

Newton's natural philosophy (philosophia naturalis) can be considered as seeking to reply to three questions. The first is this one: What are the physical objects? Newton's answer is that matter consists in particles that are distributed in a background space, a particle being a

1 See furthermore the papers in Ross, Ladyman and Kincaid (2013) for discussion as well as Ney (2012).

2 See also the balanced positions of Callender (2011) as well as Chakravartty (2013), who examines to what extent naturalized metaphysics has to go into the topics of standard metaphysics that are usually considered as being far from science. On the other end of the spectrum, see Monton (2011), whose argumentation, however, is based on the claim that our current fundamental physical theories are false. 
material object that is so small that it is localized at a point in space, thus being indivisible. Hence, some points of space are occupied - a particle is localized at them -, whereas others are empty.

If one adopts a sparse view of physical properties, there is no reason to make use of the notion of properties as far as this basic characterization of matter is concerned. Matter is primitive stuff, and it is a primitive fact that some points of space are occupied whereas others are not. There is a good reason for conceiving matter in terms of particles, that is, in terms of points of space being occupied or empty. If one considered matter to be a continuous stuff distributed all over space (that is, gunk), then one would have to maintain that there is more stuff at some points of space and less stuff at others in order to be able to accommodate variation. But it could not be a primitive fact that there is more stuff at some points of space and less at others; a property of the stuff would be needed to account for that difference. However, as we will see shortly, all the properties that classical mechanics attributes to matter concern its temporal development, not simply the fact that there is matter. The view of matter consisting in particles can easily take into account the fact that there is more matter in some regions of space than in others: in some regions of space, more points are occupied than in others.

In Newtonian mechanics, the distribution of matter in a background space develops in a background time. That is to say, as time passes, there is change in which points of space are occupied and which are empty. That change is such that the particles persist in the sense of enduring, each moving on a continuous trajectory. An alternative view would be to admit just single events, with no continuous sequences of events. But, again, taking matter to have a continuous existence - instead of events popping in a discontinuous way in and out of existence as time passes - seems to be the simpler view. Consequently, each particle has an identity in time by which it distinguishes itself from all the other particles. The particles can therefore with good reason be regarded as substances.

The fact that there is change implies that Newton has to answer a second question: What are the laws of the temporal development of the physical objects? More precisely: What are the properties of the physical objects so that certain laws describe their behaviour? Consequently, the need for a commitment to properties arises in Newton's philosophia naturalis when it comes to an account of the temporal development of the physical objects. Change in position as time passes means that the particles have the property of velocity, which is the first temporal derivative of position. That is to say, over and above having an initial position, the particles have an initial velocity, and this initial velocity makes them move in a certain manner. The property of velocity of each particle is conserved, as long as it is the only property that is taken into consideration. Velocity thereby gives rises to Newton's first law, which says that given an initial velocity, particles move on a straight line with constant velocity (inertial motion).

However, it is an empirical fact that there is not only change in the points in space that particles occupy as time passes, but also change in their state of motion, that is, change in velocity. That is why it is necessary to attribute more properties to the particles than just an initial velocity. Newton does so in taking the particles to be equipped with mass. In virtue of possessing mass, particles accelerate in the sense that they attract each other (gravitational mass) as well as resist to acceleration (inertial mass), acceleration being the change of velocity in time and thus the second temporal derivative of position. Newton's second law 
describes how properties change the state of motion of particles by accelerating them. In doing so, Newton introduces the notion of forces. Thus, in virtue of possessing mass, particles exert a force of attraction upon each other, namely the force of gravitation. However, there is no need to subscribe to an ontological commitment to forces over and above a commitment to properties of the particles such as their mass. Given the masses of the particles at a time $t$ and their positions and velocities at $t$, the acceleration of the particles at $t$ is determined (modulo the gravitational constant). Forces are a device to calculate the consequences that the presence of properties such as mass has for the change of the state of motion of the particles, but no addition to being. ${ }^{4}$ The same goes for other properties that account for the change of the state of motion of particles in classical physics, such as their charge, giving rise to acceleration due to electromagnetic interaction: there is charge determining that interaction, but no force that acts in nature over and above there being charged particles.

Finally, Newton's natural philosophy has to answer a third question: How do the physical objects and their properties explain the observable phenomena? As the quotation above shows, Newton answers this question by maintaining that (a) all macrophysical objects are composed of microphysical particles and that (b) all differences in macrophysical objects can be traced back to the position (configuration) and the change of position (motion) of the microphysical particles. That is to say, the properties that account for the temporal development of the position of the microphysical particles (that is, their initial velocity and their mass, as well as their charge) thereby also account for all the variations in the macrophysical objects.

Newton's theory is a paradigmatic example of natural philosophy in that physics and metaphysics come together in this theory in an inseparable manner. Newton's theory is not a naturalized metaphysics in the sense of being a positivist metaphysics: the assumption that there are particles and that properties of the particles have to be admitted that change the state of motion of the particles by accelerating them cannot be derived from any observation. It is an ontological postulate. But Newton's theory is not a rationalist metaphysics either: there is no a priori justification of the commitment to particles and properties that accelerate them. Making these assumptions yields a theory that is both physical-mathematical and metaphysical in one, being a universal physical theory that has the ambition to provide for a complete ontology of nature, and whose justification consists in its success in predicting and explaining the observable phenomena.

\section{Relativity physics, quantum non-locality, and the metaphysics of time}

There is, however, a stumbling block in Newton's theory, namely the assumption that particles interact instantantaneously across empty space. Maxwell's field theory of electromagnetism developed in the $19^{\text {th }}$ century provides the means to overcome this stumbling block: in virtue of being charged, particles create a field, and their interaction is transmitted by the field and thus retarded. Hence, instead of action at a distance, there is local action: interactions propagate from a space-time point to its neighbouring points. They are thereby transmitted with a finite velocity. In fact, the velocity of light is the upper limit for the propagation of effects.

4

See e.g. Jammer (1957, pp. 243-245). As regards the contemporary discussion about the ontological status of Newtonian forces, see notably Bigelow, Ellis and Pargetter (1988), Wilson (2007) and Massin (2009). 
In the special theory of relativity, Einstein (1905) draws the consequences of the field solution to the problem of action at a distance in Newtonian mechanics. This theory is built on the following two principles:

1. All inertial reference frames are equivalent for the description of physical phenomena.

2. The velocity of light is a constant, being independent of the state of motion of its source and thus the same in all inertial reference frames.

Principle (1) is taken over from pre-relativistic physics, going back to Galilei. Principle (2) implements the field solution to the problem of action at a distance in Newtonian mechanics. It implies that the Galilean transformations are no longer applicable when switching from one inertial reference frame to another one. They have to be replaced with the Lorentz transformations. The latter unify space and time in the following sense: only the fourdimensional, spatio-temporal distance between any two events occurring at space-time points is an invariant. This is the reason for the claim that following the special theory of relativity, space and time are not separate entities, but are unified in a four-dimensional space-time.

In order to draw metaphysical conclusions from these two principles, one does not have to assume that the special theory of relativity is true. Indeed, strictly speaking, it is false, since the general theory of relativity no longer treats space-time as a non-dynamical background. Nonetheless, these two principles carry over from the special to the general theory of relativity. One can therefore presume that these two principles put a constraint on any future theory of space-time, whatever the further content of such a theory may be.

These two principles suggest certain consequences for the metaphysics of time. They entail that there is no objective simultaneity, because any two events that are simultaneous in one inertial reference frame are not simultaneous in other inertial reference frames, and all inertial reference frames are equivalent; in other words, there is no unique foliation of space-time into spatial hypersurfaces that are ordered in time. Consequently, any metaphysics of time that is based on the tenses - the past, the present, the future - being objective features of the world and that ties existence to tense is incompatible with these two features.

In particular, presentism is refuted by these two principles: presentism is the view that only what is present exists. What is past no longer exists, and what is future does not exist as yet. Presentism, thus construed, takes for granted that there is a unique foliation of fourdimensional space-time into three-dimensional spatial hypersurfaces that are ordered in time. ${ }^{5}$ It is the view that these hypersurfaces come into and go out of existence such that always only one such hypersurface exists - the present one. Monton (2006, p. 264) characterizes this view as "Heraclitean presentism", because its central tenet is the reality of change in the sense of events coming into being and going out of being. Presentism thus is opposed to eternalism according to which everything that there is in nature simply exists. In the context of the special and the general theory of relativity, the latter position is known as the view of the block universe: everything that there is in space-time simply exists. Consequently, there is no temporal becoming in the sense of something coming into being as time passes.

There are strategies available to avoid drawing the metaphysical conclusion of the block universe view from relativity physics. The most prominent strategy that one can try is solipsism: if one assumes that only the space-time point at which one is situated - my "here" and "now" - exists, then no contradiction with the mentioned two principles arises (see Stein 
1968 for setting out that option and e.g. Harrington 2008 for endorsing it). But solipsism certainly is not a serious metaphysical stance based on science. Wüthrich (2013, sections 3-6) examines the various strategies envisaged in the literature to avoid the conclusion of the block universe view and convincingly argues that all these strategies are desperate. In short, taking the metaphysical position of presentism to be refuted by relativity physics and regarding eternalism in the form of the block universe view as vindicated by relativity physics is a straightforward metaphysical conclusion from space-time physics, if anything ever is a straightforward metaphysical conclusion from a scientific theory.

However, metaphysics of science is not concerned with metaphysical conclusions that one may draw from one particular scientific theory. Any metaphysics of science, whatever methodology it pursues, seeks to develop a coherent and complete vision of nature on the basis of our mature scientific theories. Thus, in order to build metaphysical conclusions on a particular scientific theory, two conditions have to be met: (a) the principles of the scientific theory in question on which the metaphysical conclusions at issue are based have to be such that we have reason to believe that these principles put a constraint on any future successor theory of the scientific theory in question. (b) The scientific theory in question either has to be itself a complete fundamental physical theory or there have to be no other contemporary mature scientific theories that challenge its principles. As argued above, condition (a) is satisfied in this case. However, condition (b) is not fulfilled: quantum physics is a mature science that has at least the same scientific standing as relativity physics. Quantum physics challenges the conjunction of the two principles on which relativity physics is based.

A popular way of setting out that challenge invokes what is known as the collapse of the wave-function in a quantum measurement process. However, whether such a collapse really occurs as a process in nature is a controversial issue, as we will see in the next section. The collapse view of measurement does not meet condition (a). There is no challenge to the block universe metaphysics stemming from wave-function collapse in quantum mechanics (see Callender 2008). Nonetheless, it is true that quantum physics calls the conjunction of the two principles on which relativity physics is based into question.

John Bell, in one of his last papers entitled "La nouvelle cuisine" (1990), formulates a principle of local causality: "The direct causes (and effects) of events are near by, and even the indirect causes (and effects) are no further away than permitted by the velocity of light" (quoted from Bell 2004, p. 239). No particular notion of causation is implied here (see Bell 2004, p. 240). The idea is that whatever events whose occurrence contributes to determining the probabilities for a given event to happen at a certain space-time point are located in the past light-cone of that event. This is one way of formulating the principle of local action that is implemented in classical field theories and that overcomes Newtonian action at a distance. Since relativity physics endorses this principle, it can waive the commitment to a unique temporal order of events and thus the commitment to an objective simultaneity: whatever contributes to determining a given event is situated in its past light cone; consequently, there is no need to settle for a unique temporal order of events that are situated outside each others light cones.

Consider the thought experiment of Einstein, Podolsky and Rosen (EPR) (1935) in the version of Bohm (1951, pp. 611-622). Two elementary quantum particles are prepared in an entangled spin state at the source of the experiment (such as two systems of spin $1 / 2$ in the singlet state). Later, when they are far apart in space so that there is no interaction any more 
between them, Alice chooses the spin parameter to measure in her wing of the experiment and obtains an outcome, and Bob does the same in his wing of the experiment. Alice's setting of her apparatus is separated by a spacelike interval from Bob's setting of his apparatus. The following figure illustrates this situation:

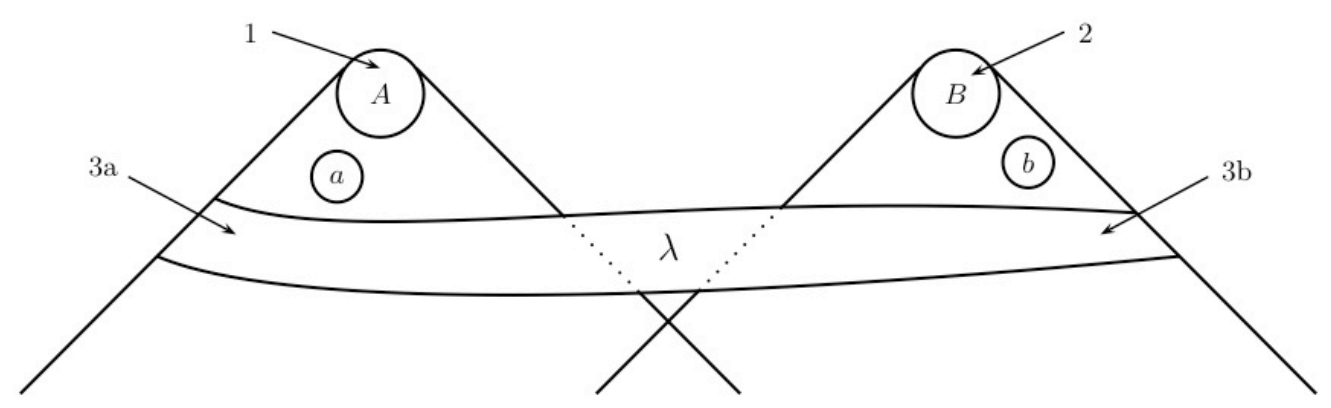

Figure 1: The situation that Bell considers in the proof of his theorem. Figure taken from Seevinck (2010, appendix) with permission of the author.

In this figure, $a$ stands for Alice's measurement setting, $A$ for Alice's outcome, $b$ stands for Bob's measurement setting, $B$ for Bob's outcome, and $\lambda$ ranges over whatever in the past may influence the behaviour of the measured quantum systems according to the theory under consideration (which may be standard quantum mechanics, or a theory that admits additional, so-called hidden variables).

Bell's principle of local causality - or locality for short - can then be formulated in the following manner:

$$
\begin{aligned}
& P_{a, b}(A \mid B, \lambda)=P_{a}(A \mid \lambda) \\
& P_{a, b}(B \mid A, \lambda)=P_{b}(B \mid \lambda)
\end{aligned}
$$

That is to say: the probabilities for Alice's outcome depend only on her measurement setting and $\lambda$. Adding Bob's setting and outcome does not change the probabilities for Alice's outcome. The same goes for Bob. The theorem that Bell proved in 1964 (reprinted in Bell 2004 , ch. 2) establishes that quantum mechanics violates (1). That is to say, for some measurement settings, even if the probabilities for Alice's outcome $A$ depend only on her setting $a$ and the past state $\lambda$, it is then necessarily so that the probabilities for Bob's outcome $B$ depend not only on his setting $b$ and the past state $\lambda$, but also on Alice's setting $a$ and outcome $A$, although $b$ and $B$ are separated by a spacelike interval from $a$ and $A$. Moreover, any theory that reproduces the well-confirmed experimental predictions of quantum mechanics has to violate (1). This conclusion applies not only to quantum mechanics, but also to quantum field theory. ${ }^{6}$ One can therefore say that Bell's theorem puts a constraint on any present or future - physical theory that is to match the experimentally confirmed predictions of quantum mechanics.

The proof of Bell's theorem does not depend on the truth of quantum mechanics or quantum field theory. The theorem then establishes that any theory that complies with the

6 See Bell (2004, ch. 24). See Hofer-Szabó and Vescernyés (2013) as well as Lazarovici (2014) for the current discussion. 
predictions for macrophysical measurement outcomes of quantum mechanics or quantum field theory - whatever its content may be - cannot satisfy the locality principle (1). One can limit the point at issue of Bell's theorem to correlations between space-like separated macrophysical measurement outcomes, such as the directions in which pointers point. In other words, even if one abstains from hypotheses about the microphysical constitution of such macrophysical events, one still gets Bell's theorem.

Nonetheless, the proof of Bell's theorem requires more than the locality principle (1): it requires also that the measurement settings $a$ and $b$ are independent of the past state $\lambda$. Failure of such independence can arise in two different ways: either the measurement settings exert some influence on $\lambda$, or $\lambda$ somehow influences the measurement settings. It is obvious from Figure 1 that the first option involves influences travelling backwards in time. Indeed, any attempt to save the locality principle (1) by relying on backwards causation retroactively correlates the measurement settings $a$ and $b$ with the past state $\lambda$ : the settings $a$ and $b$ influence the outcomes $A$ and $B$, which in turn retroactively influence $\lambda .{ }^{7}$ The second option contradicts the presupposition that the measurement settings can be freely chosen by an experimental physicist or a random generator.

However, the assumption of such an independence is not specific for Bell's theorem, but applies to any experimental evidence: if the behaviour of the measured system that produces the measurement outcome were correlated with the parameter that is measured on the system, then no conclusions about the constitution of nature would be possible on the basis of experimental evidence. Furthermore, this assumption does not imply any sort of indeterminism. A physical theory with a completely deterministic dynamics can satisfy this assumption - as does for instance Bohmian mechanics in the quantum case. ${ }^{8}$ It is therefore a well-grounded conclusion to maintain that quantum physics refutes the locality principle (1). 9

If the probabilities for what happens in a given space-time region are influenced by what happens in regions that are separated by a spacelike interval from that region, then the mentioned two principles on which the special and the general theory of relativity are built are challenged. It would, however, be unwarranted to conclude that there are signals travelling with a velocity that is much higher than the velocity of light. There is no precisely formulated version of quantum theory that includes superluminal signals, although one can contemplate models of quantum non-locality that are built on the idea of superluminal signals, as notably Chang and Cartwright (1993, section III) do. If events that occur in a space-time region that is separated from a given space-time region by a spacelike interval contribute to determining what happens in the latter region, this suggests that there is a unique or objective temporal order between these events. In other words, the principle that is challenged is the one of the equivalence of all inertial reference frames (special relativity) so that there is no unique

See Price (1996, ch. 8 and 9) for a prominent such attempt. See the papers in Studies in History and Philosophy of Modern Physics 38 (2008), pp. 705-784, for discussion.

See the exhange on this issue between Bell, Shimony, Horne and Clauser in Bell et al. (1985). The socalled free will theorem by Conway and Kochen (2006 and 2009) does not show anything standing up to scrutiny that is not already given by Bell's theorem. See notably Tumulka (2009), Goldstein et al. (2010) and Wüthrich (2011).

9 See Maudlin (2011, chs. 1-6), Norsen (2009), Seevinck (2010) and Seevinck and Uffink (2011) for the current state of the discussion on Bell's theorem. 
foliation of space-time into three-dimensional, spatial hypersurfaces that are ordered in time (general relativity).

Even if one takes the EPR-correlations between space-like separated events to require a unique temporal order of these events, one does not have to contradict any of the experimental evidence for the special and the general theory of relativity. Quantum non-locality then implies that there is more structure of space-time than is admitted by relativity physics, but this additional structure is not accessible by experience - otherwise, one could use quantum non-locality for superluminal communication. However, this is not possible. The reason is, in brief, that one cannot control the measurement outcomes $A$ and $B$ (and if there are additional, so called hidden variables in $\lambda$, one cannot have full access to these variables). The conflict between quantum physics (quantum mechanics, quantum field theory) and relativity physics (special relativity, general relativity) does not arise on the operational level, but only on the ontological level (cf. Albert 2000). Even if one regards quantum non-locality as evidence for there being a unique foliation of space-time into spatial hypersurfaces that are ordered in time, one is by no means committed to going back to endorse an ether that serves as the privileged inertial frame. On the contrary, one may contemplate the idea that the distribution of mass in the universe fixes the objective foliation of space-time, or that the universal wave-function does so (see Dürr et al. 2013b).

Introducing on the basis of quantum non-locality the assumption that there is a unique foliation of space-time rules out the inference from relativity physics to eternalism in the form of the block universe metaphysics. However, this assumption does not as such contain an argument that favours presentism over eternalism. One can even raise doubts whether this assumption is compatible with the presentism that draws its support from common sense, given notably that this unique foliation of space-time is not empirically accessible (see Callender 2008). In any case, in metaphysics of science, arguments based on common sense (or on intuitions about time, or on an a priori analysis of the concept of time) are not admissible. If one sets out to make a case for presentism, one has to develop positive arguments for this metaphysics of time based on science. The lesson of the tension between relativity physics and quantum non-locality as established by Bell's theorem is not that physics favours presentism over eternalism, or that physics is neutral with respect to this metaphysical debate, but only the following methodological one: building metaphysical conclusions on a physical theory requires spelling out how this theory can accommodate all the available evidence in its domain, and assessing this evidence involves both physics and metaphysics in an inseparable manner. I will come back to this conclusion in section 5, showing there that the stance that one takes with respect to this tension depends on one's views about laws of nature and objective modality.

\section{The problem of the referent of quantum physics}

Let us now turn to quantum mechanics. In this case, there is no straightforward answer to the question of what this theory tells us about the world, supposing that it is true or approximately true. Instead of being a theory like Newtonian mechanics in which the physics itself implements a certain ontology, we have to engage in the business of interpreting quantum mechanics, its interpretation involving to settle for a specific mathematical formulation of quantum mechanics that then enables an answer to the question of what the theory tells us 
about the world. Again, physics and metaphysics are inseparable, since metaphysical considerations determine the choice of the mathematical formulation of the physical theory.

The following, easily accessible thought experiment suggested by Einstein at the Solvay conference in Brussels in 1927 illustrates this situation (my presentation is based on de Broglie's version of the thought experiment in de Broglie 1964, pp. 28-29, and on Norsen 2005): consider a box which is prepared in such a way that there is a single elementary quantum particle in it. The box is split in two halves that are sent in opposite directions, say from Brussels to Paris and Tokyo. Suppose that Alice in Tokyo opens the box she receives and finds it to be empty. If Alice's box is empty, it then is a fact that there is a particle in the box that Bob receives in Paris.

The textbook quantum formalism represents the particle in the box by means of a wavefunction. When the box is split and the two halves are sent to Paris and to Tokyo, the wavefunction represents the particle in terms of a superposition of its being in the box that travels to Paris and its being in the box that travels to Tokyo. The operational meaning of this representation is that there is a $50 \%$ chance of finding the particle in the box that travels to Paris and a 50\% chance of finding the particle in the box that travels to Tokyo. When Alice in Tokyo opens the box she receives and finds it to be empty, this representation changes such that the wave-function represents the particle to be located in the box that travels to Paris. That sudden change is known as the collapse of the wave-function.

The problem with this formalism is the following one: if one takes the collapse of the wave-function upon measurement to represent a process that occurs in nature, one is committed to what Einstein called "spooky action at a distance" - the local operation of opening the box in Tokyo creates the fact that there is a particle in the box in Paris. If, by contrast, one takes the collapse of the wave-function upon measurement to represent an updating of information of the observer, such that before opening the box the observer does not know where the particle is, one is committed to the view that textbook quantum mechanics is an incomplete physical theory - the particle then was all the time in the box travelling to Paris, and the formalism of textbook quantum mechanics is unable to represent its trajectory. Bell's theorem then shows that one cannot complete quantum mechanics in terms of a local dynamics - that is, a dynamics complying with the locality principle (1) (although in this particular case of one particle in a box, a local account is possible and provided by de Broglie's and Bohm's quantum theory). But Bell's theorem does not settle the issue of whether or not the representation in terms of the wave-function is a complete representation of quantum objects and whether or not wave-function collapse indicates a process that occurs in nature.

Indeed, the problem of understanding quantum mechanics goes deeper than answering the question of what wave-function collapse stands for. Generally speaking, in quantum mechanics, the phase space of classical mechanics is replaced with a configuration space each point of which represents a possible configuration of particles in three-dimensional space. ${ }^{10}$ Thus, if there are $N$ particles, the configuration space has $3 N$ dimensions. On this configuration space a quantum state of the particles is defined, which can be expressed in terms of a wave-function that is a field in configuration space and that develops in time

10 See North (2013, section 2) for an argument why one should regard configuration space, and not Hilbert space, as the fundamental state space of quantum mechanics. 
according to the Schrödinger equation. This has the consequence that whenever one considers the states of two or more quantum systems, the occurrence of entangled states of these systems is generic, and their states will in general remain entangled, unless wave-function collapse occurs.

This formalism runs into the following problem of understanding: on the one hand, it seems to be committed to particles, since the dimension of the configuration space is defined by the number of particles considered. On the other hand, the law (i.e. the Schrödinger equation) is not a differential equation that is about the temporal development of a particle configuration in three-dimensional space (i.e. the development of particle positions and thus particle trajectories), but about the temporal development of a wave-function in configuration space. The fundamental problem of understanding this formalism therefore is that there is an underdetermination of what its referent is: Is it objects in ordinary space? Or is it a wavefunction in configuration space? By way of consequence, metaphysics has to come in to settle the very issue of what the formalism of quantum mechanics is about.

It is usually taken for granted that the natural world consists in matter distributed in threedimensional space or four-dimensional space-time and that the task of physics is to develop an account of matter and its temporal development (plus an account of space and time themselves). However, in a famous paper about realism in quantum mechanics, Albert (1996) claims the contrary:

... it has been essential (...) to the project of quantum-mechanical realism (in whatever particular form it takes ...) to learn to think of wave functions as physical objects in and of themselves. And of course the space those sorts of objects live in, and (therefore) the space we live in, the space in which any realistic understanding of quantum mechanics is necessarily going to depict the history of the world as playing itself out (...) is configuration space. And whatever impression we have to the contrary (whatever impression we have, say, of living in a three-dimensional space, or in a four-dimensional space-time) is somehow flatly illusory. (Albert 1996, p. 277, emphasis in the original; see also Albert 2013 as well as Ney 2011 and North 2013)

This stance is known as wave-function realism. It is motivated by attributing a literal meaning to the fact that the Schrödinger equation is about the temporal development of a wavefunction in a high-dimensional space: this stance takes that wave-function to be the object of quantum mechanics. Hence, the wave-function is an ordinary physical object, as particles or fields are ordinary physical objects in three-dimensional space in classical mechanics. The drawback of this move is that it cannot attribute a literal meaning to the fact that the dimension of the space in which the wave-function exists is defined in terms of the number of particles in three-dimensional space. Indeed, when one adopts this stance, the term "configuration space" becomes obsolete: there is no given configuration of anything that points of this space represent. The physical reality is the wave-function - to be precise, the wave-function of the universe - existing as a field in configuration space.

If one endorses this stance, the obvious task then is to develop an account of our experience of objects localized in three-dimensional space and moving in that space. In the meantime, Albert (2013) has withdrawn his claim from 1996 that doing so implies regarding our impression of living in a three-dimensional space as "somehow flatly illusory" and announces a forthcoming account of the objects of common sense in functional terms, so that from wavefunction realism one can derive common sense realism instead of having to reject the latter. 
However, as yet, such an account has not been accomplished, neither in Albert's version of wave-function realism, nor in the contemporary versions of what is known as Everettian quantum mechanics. ${ }^{11}$

Even if such an account were set out in detail, wave-function realism would not be established as simply following from the formalism of quantum mechanics. It would still require metaphysical argument to justify the conclusion that the very high dimensional space on which the wave-function of the universe is defined is the realm of physical reality, instead of three-dimensional space or four-dimensional space-time. The reason is that it is impossible to satisfy both the above mentioned elements, namely (a) that the state space of quantum mechanics is a configuration space whose dimension is determined by the number of particles existing in three-dimensional space and whose points represent possible given configurations of those particles and that (b) the fundamental law of quantum mechanics is a dynamical equation that is about the temporal development of the wave-function in that space. In other words, one cannot have both (a) a configuration space that represents a physical reality outside that space and (b) a fundamental law that is about the temporal development of an object inside that space.

Wave-function realism endorses (b) and abandons (a). The other option is to endorse (a) and to abandon (b). If one takes quantum mechanics to be about a physical reality in threedimensional space or four-dimensional space-time, one is committed to what is known as a primitive ontology of quantum mechanics (this term goes back to Dürr, Goldstein and Zanghì 2013a, ch. 2, end of section 2, originally published 1992). That ontology is primitive at least in the sense that it cannot be inferred from the formalism of quantum mechanics, but that it has to be put in as the referent of that formalism. Consequently, the fundamental law then has to be a law that describes the temporal development of the elements of the primitive ontology in three-dimensional space or four-dimensional space-time, and that law can obviously not be the Schrödinger equation (see Allori et al. 2008 for the structure of primitive ontology theories).

The de Broglie-Bohm quantum theory, going back to de Broglie (1928) and Bohm (1952) and known today as Bohmian mechanics (see the papers in Dürr, Goldstein and Zanghì 2013a) is the oldest and most widely known primitive ontology theory of quantum mechanics. Bohmian mechanics endorses particles as the primitive ontology, adding the position of the particles as additional, so-called hidden variable to the formalism of textbook quantum mechanics (so that the wave-function is not a complete representation of physical reality,

11 Wallace (2012) is the most detailed contemporary version of Everettian quantum mechanics. He rejects wave-function realism, maintaining, in brief, that the quantum state (which can be represented by the wave-function of the universe) is a state instantiated in four-dimensional space-time, developing in such a way that there are many four-dimensional space-times existing in parallel ("multiverse", "branches of the universe") (see in particular chs. 2 and 8). Nonetheless, the problem of developing an account of the experience of objects as well as ourselves being localized in one four-dimensional space-time arises in this theory in the same manner as in the one of Albert. Furthermore, Wallace's position amounts to what is known as super-substantivalism (Sklar 1974, pp. 221-224). If one maintains that physical properties or states are properties or states of space-time itself instead of being instantiated by objects localized in spacetime, thus avoiding a commitment to what is known as a primitive ontology of objects in space-time, one has to elaborate on a theory of how physical properties can be properties of space-time itself - otherwise, one only performs what Sklar (1974, pp. 166-167, 222-223) describes as a linguistic trick, namely changing language in attributing the properties that are usually ascribed to objects in space-time to spacetime itself. 
since it does not represent the actual particle positions). Consequently, there is at any time one actual configuration of particles in three-dimensional space, and the particles move on continuous trajectories in physical space. The fundamental law of Bohmian mechanics is the guiding equation, describing the temporal development of the position of the particles in three-dimensional space. The wave-function, as it figures in the guiding equation, has the job to determine the velocity of the particles at any time $t$ given their position at $t$. The Schrödinger equation then comes in as an additional law, describing how the wave-function itself develops in time.

Strictly speaking, only the universal wave-function - that is, the wave-function of the configuration of all the particles in the universe - fulfills this job: strictly speaking, the velocity of any particle at $t$ depends on the position of all the particles in the universe at $t$ via the universal wave-function. That is the way in which Bohmian mechanics takes into account the non-locality established by Bell's theorem. Nonetheless, in many situations, the position of distant particles is de facto irrelevant for the trajectory of a given particle (as in the case of the particle in Einstein's boxes). Since this theory is committed to particles moving on continuous trajectories, there is no need for wave-function collapse as a process in nature to account for measurement outcomes: these consist simply in certain particle configurations, developing according to the guiding equation. If one assumes that the initial particle configuration of the universe is typical in a precise mathematical sense, it is possible to derive in Bohmian mechanics Born's rule for the calculation of probabilities for measurement outcomes via what is known as the quantum equilibrium hypothesis (see Dür, Goldstein and Zanghì 2013a, ch. 2, originally published 1992).

Instead of subscribing to the Bohmian guiding equation, one can also go for a modification of the Schrödinger equation such that this equation includes conditions under which the wavefunction localizes spontaneously in configuration space, thus enabling it to represent objects that are localized in three-dimensional space. The most precise proposal in that respect is the one going back to Ghirardi, Rimini and Weber (GRW) (1986). However, the GRW law still is about the temporal development of the wave-function in configuration space, by contrast to a differential equation that is about the temporal development of objects in three-dimensional space. One therefore still has to put in a primitive ontology as the referent of the GRW formalism. There are two proposals in that respect developed in the literature.

The one proposal is committed to gunk in the sense of a matter density field in threedimensional space: the temporal development of the wave-function represents the temporal development of the matter density in space-time, with the spontaneous localization of the wave-function in configuration space (its collapse) representing the spontaneous contraction of gunk in certain locations so that measurement outcomes and, in general, well-localized macroscopic objects are accounted for (see Ghirardi, Grassi and Benatti 1995). Again, the dynamics is non-local, since the spontaneous contraction of gunk can occur all over space, independently of spatial distances. Thus, in the mentioned case of a particle in a box, when the box is split in two halves, the matter density of the particle strectches over both the halfboxes and spontaneously localizes in one of them upon measurement.

The other proposal is committed to single events, known as flashes, occurring at space-time points: whenever there is a spontaneous localization of the wave-function in configuration space, this collapse of the wave-function represents an event occurring in space-time, in the sense of a flash appearing centred around a space-time point. More precisely, the dynamics 
being non-local again, the collapse of the wave-function represents the spontaneous occurrence of spacelike separated, but nonetheless correlated flashes. The flash-events are all there is in space-time. Hence, there is no continuous distribution of matter in physical space, namely no trajectories or worldlines of particles, and no field - such as a matter density field - either. There only is a sparse distribution of single events in space-time (see Bell 2004, ch. 22, originally published 1987 , and Tumulka 2006).

In any case, one needs a principle or a law that establishes the link between the primitive ontology and the GRW equation such that the GRW equation can fulfill the function of describing the temporal development of the elements of the primitive ontology in threedimensional space (cf. Monton 2004). In a coherent formulation of the theory, that principle or law has to stand as the fundamental one, if the theory is to be about the temporal development of matter in three-dimensional space.

Against this background, consider the following three metaphysical claims that are often put forward as following directly from the formalism of quantum mechanics:

1) Quantum mechanics refutes the standard metaphysical view of objects, namely that objects are individuals, possessing an identity that distinguishes each object from all the other ones. The whole debate about the status of quantum particles takes for granted that the standard view is refuted and that the point at issue following quantum mechanics only is whether a notion of weak discernibility can bestow some sort of individuality on quantum particles (Saunders 2006) or whether even this is not possible (Ladyman and Bigaj 2010).

2) Since the formalism of quantum mechanics does not specify any particular objects in space-time as its referent and since interpreting quantum physics as being about objects as traditionally conceived leads to an underdetermination between an ontology of individuals and an ontology of non-individuals, quantum mechanics grounds a metaphysics of structures, known as ontic structural realism - by contrast to the object-based metaphysics that is taken for granted in mainstream analytic philosophy. This is the central claim of the naturalized metaphysics argued for by Ladyman and Ross (2007).

3) Quantum physics has implications for the metaphysics of modality: in particular, it refutes Lewis's thesis of Humean supervenience. This claim is widespread in the literature on the metaphysics of quantum physics since the seminal paper of Teller (1986). Maudlin (2007, ch. 2, pp. 51-64) turns it into a forceful attack on Humeanism in general based on quantum physics.

However, as regards the first two claims, they fail to consider the issue of what exactly the formalism of quantum mechanics represents. There is no point in seeking to draw metaphysical consequences from a formalism that contains operators which are introduced in order to allow for the calculation of probabilities of measurement outcomes - doing so would amount to what is known as naïve realism about operators (see Daumer et al. 1997). And there is no point in proposing a metaphysics of structures without considering how these structures are instantiated in the physical realm (see Esfeld 2013). Concerning the third claim, I will show in the next section that it goes through only if one presupposes an anti-Humean metaphysics of laws of nature. If, by contrast, one endorses Humeanism about laws, Lewis's thesis of Humean supervenience can be literally true even in the light of quantum physics.

The considerations in this section seek to establish that one cannot read off a metaphysics from the formalism of quantum mechanics, because the formalism as such does not specify its 
referent. The first task for naturalized metaphysics in this area therefore is to chart out the options for providing a referent for quantum mechanics. By way of consequence, what one proposes as the mathematical formulation of a fully developed quantum theory depends on metaphysical considerations. Moreover, all the known options for specifying the referent of the formalism of quantum mechanics are committed to objects, and these options cover all the traditional kinds of objects - namely particles, gunk (a matter field), and single events.

If one takes the universal wave-function to be the referent of quantum physics, then the object to which quantum physics is committed is a field, albeit a field in an extremely high dimensional space by contrast to a field in four-dimensional space-time. But the field then has definite numerical values at the points of that high-dimensional space so that these values can be regarded as intrinsic properties occurring at the points of that space. Furthermore, the dynamical law for the temporal development of this field in that space is local (as long as it is given by a linear dynamical equation such as the Schrödinger equation). Consequently, if one takes the universal wave-function to be the referent of quantum physics, one obtains a traditional field ontology with a local dynamics as in classical field theory. There hence is in this case no motivation for basing a metaphysics of structures on quantum physics (see Albert 1996, p. 283, note 7).

If, by contrast, one goes for a primitive ontology of matter distributed in ordinary spacetime as the referent of the formalism of quantum physics, then there is continuity in the space in which the physical reality plays itself out, namely four-dimensional space-time, and there is continuity in objects from classical to quantum mechanics - particles, a matter field, single events being the options for a primitive ontology of quantum mechanics as outlined above. However, there then is change in the mathematical structure of the theory from classical to quantum mechanics, since the law for the temporal development of these objects then has to be a non-local one, in order to meet the conditions set by Bell's theorem. One can then regard this non-local law as being grounded in a modal structure that takes all the physical objects as its relata and that determines their temporal development (in a deterministic or probabilistic manner) (see Esfeld 2013). Nonetheless, in any case, these physical objects come with their own identity conditions: particles and single events are absolutely discernible due to their position in physical space, and a matter field is one continuous object distributed all over physical space. In sum, there is no point in seeking to draw metaphysical conclusions about objects directly from the formalism of quantum mechanics. One first has to settle what one takes to be the referent of that formalism, and doing so brings in metaphysical considerations. Once this has been done, there then is no longer an issue about the discernibility or the identity conditions of quantum objects.

\section{Physics and the metaphysics of modality}

Let us assume that the referent of the formalism of quantum mechanics is the distribution of matter in ordinary space-time. It then seems obvious that entanglement shows that quantum mechanics refutes Humean metaphysics, in particular David Lewis's thesis of Humean supervenience:

It is the doctrine that all there is to the world is a vast mosaic of local matters of particular fact, just one little thing and then another. (...) We have geometry: a system of external relations of spatio-temporal distance between points. ... And at those points we have local qualities: perfectly natural intrinsic properties which need nothing bigger than a point at which to be 
instantiated. For short: we have an arrangement of qualities. And that is all. ... All else supervenes on that. (Lewis 1986, pp. ix-x)

It seems evident that Lewis's view of the supervenience basis, consisting exclusively in the distribution of intrinsic physical properties located at space-time points, is contradicted by the fact of there being relations of quantum entanglement (see Teller 1986 and for a recent statement of this view Humphreys 2013, pp. 56-57). Notably Maudlin (2007, ch. 2, pp. 51-64) argues that quantum entanglement (the non-separability of quantum states) refutes not only Lewis's conception of the supervenience basis, but thereby also the Humean rejection of objective modality. The formalism of quantum physics thus has a direct bearing not only on what there is in the fundamental physical domain of the actual world, but also on which philosophical views of modality - and thereby of laws of nature - are admissible.

Lewis's reason for proposing a metaphysics of fundamental physics that recognizes only intrinsic properties located at space-time points is free combinatorialism: one can hold any local quality occurring at a space-time point fixed and vary all the other local qualities, the result always is a possible world. Any property occurrences can be combined with any other property occurrences, no instantiation of a property poses any restrictions on what the world has to be like beyond the space-time point at which the property in question is instantiated. Hence, there are no necessary connections in the world.

Philosophers with a favourable attitude towards Humeanism reacted to the challenge from quantum physics by trying to adapt Humeanism so that quantum entanglement is taken into account. The most important suggestion in this respect is to admit irreducible relations of entanglement over and above the spatio-temporal relations to the ontological ground floor of Humeanism (Darby 2012) and to envisage developing a Humean version of ontic structural realism on the basis of including such relations (Lyre 2010). However, recognizing irreducible relations of quantum entanglement considerably restricts free combinatorialism and arguably implies a commitment to some sort of objective modality, since these relations tie the temporal development of - in the last resort all - quantum systems together, whatever their spatial distance may be. Thus, considering the EPR experiment with the same parameter measured in both wings of the experiment, if in one wing the measured quantum system behaves in such a way that the measurement outcome is spin up, then it is necessarily so that in the other wing the measured quantum system behaves in such a way that the measurement outcome is spin down. If one does not want to talk about two systems in this respect, one can also formulate this point by saying that if in one wing of the experiment the pointer of the measuring apparatus indicates the outcome spin up, then it is necessarily so that in the other wing of the experiment the pointer of the measuring apparatus indicates the outcome spin down. Consequently, in any case, if there are relations of quantum entanglement in the supervenience basis, these relations pose a constraint on what can and what cannot happen elsewhere in space-time.

Furthermore, one can adapt Humeanism to quantum physics by adopting wave-function realism and admitting the very high-dimensional configuration space of the universe instead of four-dimensional space-time as the realm of physical reality (Loewer 1996). In this case, as mentioned at the end of the last section, everything is local in that space. Nonetheless, it is a considerable change of Humean metaphysics, which is inspired by common sense realism, to switch to configuration space as the stage of the Humean supervenience basis. 
However, in recent years, it has become clear that no such adaptation is necessary. Humeanism is not refuted by quantum physics. More precisely, Lewis's thesis of Humean supervenience can be literally true even in the light of the empirical evidence for quantum entanglement. The background that enables Humeanism to stand firm is the development of primitive ontology theories of quantum physics, as outlined in the preceding section. To recap, the primitive ontology consists in the distribution of matter in three-dimensional space or four-dimensional space-time; that distribution is the referent of the formalism of quantum physics. Furthermore, a law is admitted as that what fixes (in a probabilistic or a deterministic manner) the temporal development of the distribution of matter in physical space, given an initial configuration of matter. That's all. In particular, according to the primitive ontology theories, the quantum mechanical wave-function is part and parcel of the law instead of being a physical entity on a par with the primitive ontology.

Since the primitive ontology is in any case constituted by local matters of particular fact "local beables" to use Bell's famous term (Bell 2004, ch. 7) -, the only move that the Humean has to make is this: instead of admitting the law as an entity that exists in addition to and independently of the primitive ontology, governing or guiding the temporal development of the primitive ontology, the Humean has to regard the law as supervening on the distribution of matter throughout the whole of space-time, that is, the entire mosaic of "local beables" or local matters of particular fact. This move has been made with respect to Bohm's quantum theory in recent literature (see Callender unpublished, Esfeld et al. 2014, section 3, Miller 2014). It is obvious that it can be extended also to the GRW matter density ontology and the GRW flash ontology (see Callender 2015 and Esfeld 2014). It is no objection to this move that the quantum mechanical wave-function does not supervene on the configuration of matter in space at any given time, since the Humean claims only that it supervenes on the entire distribution of matter in the whole of space-time. In a nutshell, on the Humean view, the universal wave-function is fixed only at the end of the world. If the entire distribution of matter in space-time were still to leave room for different universal wave-functions, that difference would not make any empirical difference and could therefore be dismissed by the Humean as a mathematical surplus structure.

Indeed, already Bell himself recognized this position as a coherent stance in the paper in which he introduced the notion of "local beables" (1975) ("beable" is Bell's neologism for what exists by contrast to "observable", that is, what can be observed):

One of the apparent non-localities of quantum mechanics is the instantaneous, over all space, 'collapse of the wave function' on 'measurement'. But this does not bother us if we do not grant beable status to the wave function. We can regard it simply as a convenient but inessential mathematical device for formulating correlations between experimental procedures and experimental results, i.e., between one set of beables and another. (Quoted from Bell 2004, p. 53)

Bell makes two points in this quotation: (1) It is not mandatory to grant beable status to the wave-function. If one admits "local beables", one has an ontology of the physical world. Not granting beable status to the wave-function does, however, not commit one to an instrumentalist attitude to the wave-function, as Bell suggests here. Humeanism is distinct from instrumentalism (Miller 2014, section 5, stresses this point). The Humean only has to maintain that the primitive ontology is the full ontology, with everything else supervening on it. That is why Humeanism is also not touched by recent claims about experimental evidence 
in favour of the reality of the wave-function (Pusey, Barrett and Rudolph 2012; Colbeck and Renner 2012): these claims challenge only the view that the wave-function represents nothing but the information about probabilities for measurement outcomes that is available for an observer. However, on Humeanism, the universal wave-function is not relative to observers: it is an objective matter of fact in supervening on the entire distribution of the "local beables". Since any experimental evidence consists in "local beables", the Humean is in the position to accommodate whatever experimental evidence there may be.

(2) Given that it is the wave-function which is entangled and which correlates "local beables" whatever their spatial or spatio-temporal distance is, if one does not grant beable status to the wave-function, there is no reason to admit non-supervenient relations of entanglement (or of dependence or of influence) among the "local beables" over and above their occurrence at space-time points. In being entangled, the wave-function establishes such correlations, but these are no addition to what there is over and above the occurrence of the "local beables" at space-time points, since the universal wave-function and its temporal development supervene on the entire mosaic of these "local beables". Hence, even if one regards ordinary space-time as the realm of physical reality also in quantum physics, if one adopts Humeanism, there is again no motivation to go for ontic structural realism based on quantum physics.

By way of consequence, if one takes the primitive ontology to be the full ontology, there is no problem with Lorentz-invariance, since the primitive ontology consists entirely in local matters of particular fact. However, as soon as one takes the entanglement of the wavefunction in configuration space to refer to dependency relations among some such local matters of particular fact over and above their simple occurrence at space-time points, then Bell's theorem implies that there is no Lorentz-invariant theory of these dependency relations or influences possible: there then is a fact for any given flash-event, particle position or matter density value at a space-time point of whether or not the occurrence of that flash, particle position or matter density value depends on where other flash-events, particle positions or matter density values occur at spacelike separated locations. This conclusion is not called into question by the work of Tumulka (2006 and 2009) who has shown for the GRW flash ontology that the GRW law is Lorentz-invariant and that there is no problem with Lorentzinvariance as long as one considers only probabilities for entire distributions of flashes in space-time; 12 this important result does not touch upon the fact that for the occurrence of each new flash, it remains a meaningful question to ask whether or not the occurrence of that flash depends on or is influenced by where at a spacelike separated location other flashes occur, and there is no Lorentz-invariant answer to that question available (see Esfeld and Gisin 2014). Consequently, the metaphysical issue of Humeanism vs. anti-Humeanism about laws of nature and objective modality has direct implications for the issue of whether or not a Lorentz-invariant quantum ontology of matter distributed in space-time is available. For the Humean, it is no problem to obtain such an ontology, whereas for the anti-Humean, such an ontology is not available.

Since the Humean does not grant beable status to the wave-function, there is nothing that determines the temporal development of an initial configuration of "local beables". The particle positions simply happen to develop in such a way that there are, as far as Bohmian

12 Bedingham et al. (2014) seek to achieve a similar result for the GRW matter density field ontology. 
quantum mechanics is concerned, continuous particle trajectories; the matter density values just happen to develop in such a way that the matter density takes a certain shape making true the GRW law, and the flash-events just happen to occur in such a manner that they make true a law of the GRW type. There is nothing that drives, guides or forces them to do so. This is simply what the general Humean attitude towards laws and objective modality implies. One may have reservations about that attitude. But there is nothing in quantum physics that obliges one to abandon it. In brief, it is "anti-Humeanism in, anti-Humeanism out", or "Humeanism in, Humeanism out". If one assumes that the wave-function is some sort of a real entity over and above the primitive ontology, then quantum physics comes out anti-Humean. If, by contrast, one bases oneself on the empiricist idea that the primitive ontology is the full ontology, then one obtains a Humean ontology of quantum physics.

This is not to say that physics has no bearing on the metaphysics of modality. One important argument in this respect is that Humeanism cannot explain why the regularities of physics - such as the law of gravitation - always turn out to be well-confirmed (and thus, for instance, why a human being cannot fly into the air without technical aid). On Humeanism, there is no constraint at all on which local matters of particular fact can and which ones cannot occur in the future of any given local matter of particular fact; the laws of nature supervene only on the mosaic of the local matters of particular fact in the whole of spacetime. Hence, what the laws of nature are depends on what there will happen in the future of any given local matter of particular fact, instead of that future depending on the laws of nature. Dispositional essentialism, the most prominent contemporary form of anti-Humeanism about laws of nature, by contrast, provides for such an explanation: to take up the example of the law of gravitation, if it is essential for the property of mass to exercize a causal role as described by the law of gravitation (whatever the correct law of gravitation may be), then this is the reason why the regularities of physics concerning gravitation always turn out to be wellconfirmed (and thus why it would be futile for a human being to try to fly into the air without technical aid) (see notably Bird 2007). The occurrence of mass then poses a constraint on what there can be in the future of any given such occurrence. By the same token, turning to quantum physics, dispositional essentialism (or modal ontic structural realism in this case) can and Humeanism cannot explain why the outcomes of an EPR type experiment always turn out to be correlated (namely because there is a dispositional property or modal structure of entanglement instantiated by the configuration of objects in physical space). ${ }^{13}$ However, an argument of this type is a general argument from physics against Humeanism, and not a refutation of Humeanism by a particular physical theory.

Against this background, let us reconsider classical mechanics and draw some general conclusions. As pointed out in section 1, the commitment to properties of the primitive matter - that is, the particles - arises in Newton's theory, because there is something that plays a causal role in the temporal development of the trajectories of the particles. Thus, in virtue of possessing mass, the particles accelerate each other. Referring to the property of gravitational mass instantiated by the particles provides for a causal explanation of the acceleration of the particles independently of whether or not a medium is indicated by means of which the influence that particles exert on each other's state of motion is transmitted and independently 
of whether or not time passes between the presence of gravitational mass (the cause) and the acceleration of the particles (the effect).

In this vein, Blondeau and Ghins (2012) argue that the "general form of a causal law is an equation that exhibits the following mathematical form:

$$
E=\partial x / \partial t=C_{1}+\ldots C_{\mathrm{n}}
$$

$E$ refers to the effect, whereas the causes $C_{\mathrm{i}}$ can, but need not, be functions of time. The above general form reads: $C_{1}, C_{2}, \ldots$ are the causes of the infinitesimal variation of the property $x$ of a system, i.e. of the effect $E^{\prime \prime}$ (p. 384). The decisive point is that any law fitting into this form is asymmetric in that what appears on the right side induces a certain temporal development of the quantity on the left side, but not vice versa, without any time having to pass between the presence of the causes $C_{1} \ldots C_{\mathrm{n}}$ and the effect $E$, that is, the manner in which $x$ develops in time. Thus, on Newton's law of gravitation, the presence of gravitational mass induces a change in the velocity of the particles without any time passing between the presence of mass and the acceleration of the particles.

For the Humean, the causal role that the properties referred to by " $C_{1}+\ldots C_{\mathrm{n}}$ " in the formula above exercize is a contingent one: it varies from one possible world to another, supervening on the distribution of the local matters of particular fact in the world in question as a whole. Thus, it is contingent that the property we refer to as "mass" exercizes the role expressed in Newton's law of gravitation in the actual world (assuming, for the sake of the argument, that Newton's law is the correct law of gravitation for the actual world). The antiHumean, by contrast, does not regard that causal role as contingent. For the dispositional essentialist, properties are dispositions whose essence it is to exercize a certain causal role, such that whenever the property in question is instantiated in a possible world, it exercizes the same role in any world. The law expresses that role.

Hence, as far as classical mechanics is concerned, the Humean and the anti-Humean can both agree that the matter distributed in space-time (i.e. the particles) instantiates certain properties such as mass, or charge. Their dispute concerns the issue of whether or not the causal role that these properties exercize according to the laws of classical mechanics (fulfilling the scheme indicated above) is contingent or necessary (essential) to them. However, when it comes to quantum physics, the Humean can no longer recognize such properties: whereas mass and charge can be considered as intrinsic properties of particles "which need nothing bigger than a point at which to be instantiated" (Lewis 1986, p. x), there are no such intrinsic properties as far as the features that are specific for quantum physics are concerned. If one seeks for properties in quantum physics that fill in the scheme provided by formula (2) above, these can only be relations or structures of entanglement, relating all the objects in physical space (be it particles, a matter density field, or flashes in the sense of single events). Thus, the dispositional essentialist can regard the quantum mechanical wavefunction as referring to a dispositional property or modal structure instantiated by the configuration of matter as a whole and determining (in a deterministic or probabilistic manner) the temporal development of the configuration of matter. ${ }^{14}$ But the Humean cannot admit relations or structures of entanglement on pain of destroying Humean supervenience.

14 See Dorato and Esfeld (2010) for dispositionalism about the GRW ontologies. For dispositionalism about Bohmian mechanics, see Belot (2012, pp. 77-80) and Esfeld et al. (2014, sections 4-5). 
Consequently, quantum physics does after all have a repercussion for Lewis's Humean ontology: in the light of quantum physics, one can no longer maintain that the mosaic of local matters of particular fact consists in "local qualities: perfectly natural intrinsic properties which need nothing bigger than a point at which to be instantiated" (Lewis 1986, p. x). There are no such qualities or intrinsic properties in quantum physics. Quantum entanglement rules out that such local, intrinsic properties could do any work as far as the features that are specific for quantum physics are concerned. Nonetheless, quantum entanglement notwithstanding, Lewis's thesis of Humean supervenience can be literally true in quantum physics, as the primitive ontology theories show. The only adaptation that is necessary to obtain this result is to consider the mosaic of local matters of particular fact as being constituted by primitive stuff distributed in space-time. That stuff can be particles or flashes, with a particle or a flash being at a space-time point signifying that there is stuff located at the point instead of the point being empty, or that stuff can be gunk in the sense of a continuous matter density field.

The option to maintain that the matter distributed in space-time and constituting the Humean supervenience basis consists in primitive stuff instead of local qualities (intrinsic properties) is not limited to quantum physics, but also available for classical mechanics. Also with respect to classical mechanics, one can subscribe to the view that mass and charge, like the quantum mechanical wave-function, are only variables that appear in the best system, that is, the system that achieves the best balance between being simple and being informative in describing the distribution of local matters of particular fact - such as particle positions throughout the whole of space-time (see Hall unpublished §5.2). In other words, there are not mass and charge instantiated as intrinsic properties occurring at space-time points over and above particle positions signifying that a space-time point is occupied by stuff instead of being empty, as there is no wave-function instantiated as a relation or structure in space-time over and above the elements of whatever may be the primitive ontology of quantum physics. Adopting this stance removes the stock objections against Humeanism from quidditism and humility: if there were intrinsic properties instantiated at space-time points that exercize a causal role contingently, their essence would be a pure quality (a quiddity) to which we could moreover have no epistemic access (humility) (see Lewis 2009). ${ }^{15}$ Again, it is evident that the relationship between physics and metaphysics goes in both directions, with the physics here shaping Humean metaphysics in such a way that a central metaphysical objection against Humeanism no longer applies.

In conclusion, this chapter has sought to show the following:

a) how physics and metaphysics match in Newton's philosophia naturalis;

b) how even what seems to be a clear-cut case of metaphysical conclusions following directly from the formalism of a physical theory (presentism being ruled out by special

15 There also is a sort of humility implied by the primitive ontology theories of quantum mechanics: in order for these theories to make the right empirical predictions and to rule out exploiting quantum non-locality for superluminal signalling, they have to limit the epistemic access that we can have to the elements of the primitive ontology in the sense that we cannot know the exact initial conditions (that is, the exact initial particle configuration in Bohmian mechanics, the exact initial matter density distribution in the GRW matter density ontology, or the exact initial configuration of flashes in the GRW flash ontology). However, this is not an ignorance of the types of properties or entities that there are in the actual world, but only an ignorance of initial conditions, albeit a principled one. 
relativity) is called into question when one takes the whole of contemporary fundamental physics into account;

c) how specifying what the very referent of the formalism of quantum mechanics is draws on metaphysical considerations;

d) how the stance that one takes in the metaphysics of laws and modality shapes the options that are available for an ontology of quantum physics.

In sum, far from separating physics from metaphysics, the physics of the $20^{\text {th }}$ century calls for natural philosophy in the sense of an enterprise that regards physics and metaphysics as forming a seamless whole in the enquiry into the constitution of the world, at least as much as the physics of the $17^{\text {th }}$ and the $18^{\text {th }}$ century did.

\section{References}

Albert, David Z. (1996): "Elementary quantum metaphysics". In: J. T. Cushing, A. Fine and S. Goldstein (eds.): Bohmian mechanics and quantum theory: An appraisal. Dordrecht: Kluwer. Pp. 277-284.

Albert, David Z. (2000): "Special relativity as an open question". In: H.-P. Breuer and F. Petruccione (eds.): Relativistic quantum measurement and decoherence. Berlin: Springer. Pp. 1-13.

Albert, David Z. (2013): "Wave function realism". In: D. Albert and A. Ney (eds.): The wave function: essays on the metaphysics of quantum mechanics. Oxford: Oxford University Press. Pp. 52-57.

Allori, Valia, Goldstein, Sheldon, Tumulka, Roderich and Zanghì, Nino (2008): "On the common structure of Bohmian mechanics and the Ghirardi-Rimini-Weber theory". British Journal for the Philosophy of Science 59, pp. 353-389.

Bedingham, Daniel, Dürr, Detlef, Ghirardi, Gian Carlo, Goldstein, Sheldon, Tumulka, Roderich and Zanghì, Nino (2014): "Matter density and relativistic models of wave function collapse". Journal of Statistical Physics 154, pp. 623-631.

Bell, John S. (2004): Speakable and unspeakable in quantum mechanics. Cambridge: Cambridge University Press. Second edition. First edition 1987.

Bell, John S., Shimony, Abner, Horne, Michael A. and Clauser, John F. (1985): "An exchange on local beables". Dialectica 39, pp. 85-110.

Belot, Gordon (2012): "Quantum states for primitive ontologists. A case study". European Journal for Philosophy of Science 2, pp. 67-83.

Bigelow, John, Ellis, Brian and Pargetter, Robert (1988): "Forces". Philosophy of Science 55, pp. 614-630.

Bird, Alexander (2007): Nature's metaphysics. Laws and properties. Oxford: Oxford University Press.

Blondeau, Julien and Ghins, Michel (2012): "Is there an intrinsic criterion for causal lawlike statements?" International Studies in the Philosophy of Science 26, pp. 381-401.

Bohm, David (1951): Quantum theory. Englewood Cliffs: Prentice-Hall.

Bohm, David (1952): "A suggested interpretation of the quantum theory in terms of 'hidden' variables". Physical Review 85, pp. 166-193.

Callender, Craig (2008): "Finding 'real' time in quantum mechanics". In: W. L. Craig and Q. Smith (eds.): Einstein, relativity, and absolute simultaneity. London: Routledge. Pp. 50-72.

Callender, Craig (2011): "Philosophy of science and metaphysics". In: S. French and J. Saatsi (eds.): The Continuum companion to the philosophy of science. London: Continuum. Pp. 33-54.

Callender, Craig (unpublished): "Discussion: the redundancy argument against Bohm's theory". Manuscript. http://philosophyfaculty.ucsd.edu/faculty/ccallender/publications.shtml

Callender, Craig (2015): “One world, one beable”. Synthese 192, pp. 3153-3177.

Chakravartty, Anjan (2013): "On the prospects of naturalized metaphysics". In: D. Ross, J. Ladyman and H. Kincaid (eds.): Scientific metaphysics. Oxford: Oxford University Press. Pp. 27-50.

Chang, Hasok and Cartwright, Nancy (1993): "Causality and realism in the EPR experiment". Erkenntnis 38, pp. 169-190. 
Colbeck, Roger and Renner, Renato (2012): "Is a system's wave function in one-to-one correspondence with its elements of reality?" Physical Review Letters 108, 150402.

Conway, John H. and Kochen, Simon (2006): "The free will theorem”. Foundations of Physics 36, pp. 14411473.

Conway, John H. and Kochen, Simon (2009): "The strong free will theorem". Notices of the American Mathematical Society 56, pp. 226-232.

Darby, George (2012): "Relational holism and Humean supervenience". British Journal for the Philosophy of Science 63, pp. 773-788.

Daumer, Martin, Dürr, Detlef, Goldstein, Sheldon and Zanghì, Nino (1996): "Naive realism about operators". Erkenntnis 45, pp. 379-397.

de Broglie, Louis (1928): "La nouvelle dynamique des quanta". In: Electrons et photons. Rapports et discussions du cinquième Conseil de physique tenu à Bruxelles du 24 au 29 octobre 1927 sous les auspices de l'Institut international de physique Solvay. Paris: Gauthier-Villars. Pp. 105-132. English translation in G. Bacciagaluppi and A. Valentini (2009): Quantum theory at the crossroads. Reconsidering the 1927 Solvay conference. Cambridge: Cambridge University Press. Pp. 341-371.

de Broglie, Louis (1964): The current interpretation of wave mechanics. A critical study. Amsterdam: Elsevier.

Dorato, Mauro and Esfeld, Michael (2010): "GRW as an ontology of dispositions". Studies in History and Philosophy of Modern Physics 41, pp. 41-49.

Dürr, Detlef, Goldstein, Sheldon and Zanghì, Nino (2013a): Quantum physics without quantum philosophy. Berlin: Springer.

Dürr, Detlef, Goldstein, Sheldon, Norsen, Travis, Struyve, Ward and Zanghì, Nino (2013b): "Can Bohmian mechanics be made relativistic?" Proceedings of the Royal Society A 470, p. 2162.

Einstein, Albert (1905): “Zur Elektrodynamik bewegter Körper”. Annalen der Physik 17, pp. 891-921.

Einstein, Albert, Podolsky, Boris and Rosen, Nathan (1935): "Can quantum-mechanical description of physical reality be considered complete?" Physical Review 47, pp. 777-780.

Esfeld, Michael (2013): "Ontic structural realism and the interpretation of quantum mechanics". European Journal for Philosophy of Science 3, pp. 19-32.

Esfeld, Michael (2014): "Quantum Humeanism, or: physicalism without properties". Philosophical Quarterly 64, pp. 453-470.

Esfeld, Michael and Gisin, Nicolas (2014): "The GRW flash theory: a relativistic quantum ontology of matter in space-time?". Philosophy of Science 81, pp. 248-264.

Esfeld, Michael, Lazarovici, Dustin, Hubert, Mario and Dürr, Detlef (2014): "The ontology of Bohmian mechanics". British Journal for the Philosophy of Science 65, pp. 773-796.

Fine, Kit (2005): "Tense and reality". In: K. Fine (ed.): Modality and tense: philosophical papers. Oxford: Oxford University Press. Pp. 261-320.

Ghirardi, Gian Carlo, Grassi, Renata and Benatti, Fabio (1995): "Describing the macroscopic world: closing the circle within the dynamical reduction program". Foundations of Physics 25, pp. 5-38.

Ghirardi, Gian Carlo, Rimini, Alberto and Weber, Tullio (1986): "Unified dynamics for microscopic and macroscopic systems". Physical Review D 34, pp. 470-491.

Goldstein, Sheldon, Tausk, Daniel V., Tumulka, Roderich and Zanghì, Nino (2010): "What does the free will theorem actually prove?" Notices of the American Mathematical Society 57 (11), pp. 1451-1453.

Hall, Ned (unpublished): "Humean reductionism about laws of nature". http://philpapers.org/rec/HALHRA

Harrington, James (2008): "Special relativity and the future: a defense of the point present". Studies in History and Philosophy of Modern Physics 39, pp. 82-101.

Hofer-Szabó, Gábor and Vecsernyés, Péter (2013): "Bell inequality and common causal explanation in algebraic quantum field theory". Studies in History and Philosophy of Modern Physics 44, pp. 404-416.

Humphreys, Paul (2013): "Scientific ontology and speculative ontology". In: D. Ross, J. Ladyman and H. Kincaid (eds.): Scientific metaphysics. Oxford: Oxford University Press. Pp. 51-78.

Jackson, Frank (1998): From metaphysics to ethics. A defence of conceptual analysis. Oxford: Oxford University Press. 
Jammer, Max (1957): Concepts of force: a study in the foundations of dynamics. Cambridge (Massachusetts): Harvard University Press.

Ladyman, James and Bigaj, Tomasz F. (2010): "The principle of the identity of indiscernibles and quantum mechanics". Philosophy of Science 77, pp. 117-136.

Ladyman, James and Ross, Don (2007): Every thing must go. Metaphysics naturalized. Oxford: Oxford University Press.

Lazarovici, Dustin (2014): "Lost in translation. A comment on 'Noncommutative causality in algebraic quantum field theory"'. In: M. C. Galavotti et al. (eds.): New directions in the philosophy of science. The philosophy of science in a European perspective. Volume 5. Cham: Springer. Pp. 555-560.

Lewis, David (1986): Philosophical papers. Volume 2. Oxford: Oxford University Press.

Lewis, David (2009): "Ramseyan humility". In: D. Braddon-Mitchell and R. Nola (eds.): Conceptual analysis and philosophical naturalism. Cambridge (Massachusetts): MIT Press. Pp. 203-222.

Loewer, Barry (1996): "Humean supervenience”. Philosophical Topics 24, pp. 101-127.

Lyre, Holger (2010): "Humean perspectives on structural realism". In: F. Stadler (ed.): The present situation in the philosophy of science. Dordrecht: Springer. Pp. 381-397.

Massin, Olivier (2009): “The metaphysics of forces". Dialectica 63, pp. 555-589.

Maudlin, Tim (2007): The metaphysics within physics. Oxford: Oxford University Press.

Maudlin, Tim (2011): Quantum non-locality and relativity. Third edition. Chichester: Wiley-Blackwell.

Maudlin, Tim (2012): Philosophy of physics. Volume 1. The arena: space and time. Princeton: Princeton University Press.

Miller, Elizabeth (2014): "Quantum entanglement, Bohmian mechanics, and Humean supervenience". Australasian Journal of Philosophy 92, pp. 567-583.

Monton, Bradley (2004): "The problem of ontology for spontaneous collapse theories". Studies in History and Philosophy of Modern Physics 35, pp. 407-421.

Monton, Bradley (2006): "Presentism and quantum gravity". In: D. Dieks (ed.): The ontology of spacetime. Amsterdam: Elsevier. Pp. 263-280.

Monton, Bradley (2011): "Prolegomena to any future physics-based metaphysics". In: J. Kvanvig (ed.): Oxford Studies in philosophy of religion. Volume III. Oxford: Oxford University Press. Pp. 142-165.

Newton, Isaac (1952): Opticks or a treatise of the reflections, refractions, inflections and colours of light. Edited by I. B. Cohen. New York: Dover.

Ney, Alyssa (2011): "The status of our ordinary three dimensions in a quantum universe". Noûs 44, pp. 1-36.

Ney, Alyssa (2012): "Neo-positivist metaphysics". Philosophical Studies 160, pp. 53-78.

Norsen, Travis (2005): "Einstein's boxes”. American Journal of Physics 73, pp. 164-176.

Norsen, Travis (2009): "Local causality and completeness: Bell vs. Jarrett”. Foundations of Physics 39, pp. 273294.

North, Jill (2013): “The structure of a quantum world". In: D. Albert and A. Ney (eds.): The wave function: essays on the metaphysics of quantum mechanics. Oxford: Oxford University Press. Pp. 184-202.

Price, Huw (1996): Time's arrow and Archimedes' point. New directions for the physics of time. Oxford: Oxford University Press.

Pusey, Matthew F., Barrett, Jonathan and Rudolph, Terry (2012): "On the reality of the quantum state". Nature Physics 8, pp. 475-478.

Ross, Don, Ladyman, James and Kincaid, Harold (eds.) (2013): Scientific metaphysics. Oxford: Oxford University Press.

Saunders, Simon (2006): “Are quantum particles objects?” Analysis 66, pp. 52-63.

Seevinck, Michiel P. (2010): "Can quantum theory and special relativity peacefully coexist? Invited white paper for Quantum Physics and the Nature of Reality, John Polkinghorne 80th Birthday Conference. St Annes College, Oxford. 26 - 29 September 2010". http://arxiv.org/abs/1010.3714. 
Seevinck, Michiel P. and Uffink, Jos (2011): "Not throwing out the baby with the bathwater: Bell's condition of local causality mathematically 'sharp and clean"'. In: D. Dieks, W. Gonzalez, S. Hartmann, T. Uebel and M. Weber (eds.): Explanation, prediction and confirmation. New trends and old ones reconsidered. Dordrecht: Springer. Pp. 425-450.

Sklar, Lawrence (1974): Space, time and spacetime. Berkeley: University of California Press.

Teller, Paul (1986): "Relational holism and quantum mechanics". British Journal for the Philosophy of Science 37, pp. 71-81.

Tumulka, Roderich (2006): "A relativistic version of the Ghirardi-Rimini-Weber model". Journal of Statistical Physics 125, pp. 825-844.

Tumulka, Roderich (2007): “Comment on The free will theorem”. Foundations of Physics 34, pp. 186-197.

Tumulka, Roderich (2009): "The point processes of the GRW theory of wave function collapse". Reviews in Mathematical Physics 21, pp. 155-227.

Wallace, David (2012): The emergent multiverse. Quantum theory according to the Everett interpretation. Oxford: Oxford University Press.

Wilson, Jessica (2007): "Newtonian forces". British Journal for the Philosophy of Science 58, pp. 173-205.

Wüthrich, Christian (2011): "Can the world be shown to be indeterministic after all?" In: C. Beisbart and S. Hartmann (eds.): Probabilities in physics. Oxford: Oxford University Press. Pp. 365-390.

Wüthrich, Christian (2013): "The fate of presentism in modern physics". In: R. Ciunti, K. Miller and G. Torrengo (eds.): New papers on the present - focus on presentism. München: Philosophia. Pp. 91-131. 